\title{
Morita equivalence of multidimensional noncommutative tori.
}

\author{
Marc A. Rieffel and Albert Schwarz * \\ Department of Mathematics, UC Berkeley, Berkeley, CA 94720-3840 \\ Department of Mathematics, UC Davis, Davis, CA 95616
}

April 10, 1998

\begin{abstract}
One can describe an $n$-dimensional noncommutative torus by means of an antisymmetric $n \times n$ matrix $\theta$. We construct an action of the group $S O(n, n \mid \mathbf{Z})$ on the space of $n \times n$ antisymmetric matrices and show that, generically, matrices belonging to the same orbit of this group give Morita equivalent tori. Some applications to physics are sketched.
\end{abstract}

By definition [R5, an $n$-dimensional noncommutative torus is an associative algebra with involution having unitary generators $U_{1}, \ldots, U_{n}$ obeying the relations

$$
U_{k} U_{j}=e\left(\theta_{k j}\right) U_{j} U_{k},
$$

where $e(t)=e^{2 \pi i t}$ and $\theta$ is an antisymmetric matrix. The same name is used for different completions of this algebra. In particular, we can consider the noncommutative torus as a $C^{\star}$-algebra $A_{\theta}$ (the universal $C^{\star}$-algebra generated by $n$ unitary operators satisfying (1) ). Noncommutative tori are important in many problems of mathematics and physics. It was shown recently that they are essential in consideration of compactifications of M(atrix) theory ([CDS]; for further development see [T]). The results of the present paper also have application to physics.

If two algebras $\mathcal{A}$ and $\hat{\mathcal{A}}$ are Morita equivalent (see the definition below), then for every $\mathcal{A}$-module $R$ one can construct an $\hat{\mathcal{A}}$-module $\hat{R}$ in such a way that the correspondence $R \rightarrow \hat{R}$ is an equivalence of categories of $\mathcal{A}$-modules and $\hat{\mathcal{A}}$-modules.

* M. A. R. supported in part by NSF grant DMS 96-13833; A. S. supported in part by NSF grant DMS 95-00704; both authors supported in part by NSF Grant No. PHY94-07194 during visits to the Institute for Theoretical Physics, Santa Barbara. A.S. acknowledges also the hospitality of MIT, IAS and Rutgers University 
It was shown in CDS that in the consideration of toroidal compactification of $\mathrm{M}$ (atrix) theory one can identify states with connections on projective $\mathcal{A}$ modules, where $\mathcal{A}$ is a noncommutative torus.

One can verify that compactifications on Morita equivalent tori are in some sense physically equivalent; this fact is conjectured in [CDS] and proved in [S].

In particular, it is shown in [S] that there is a correspondence between BPS states on the module $R$ with BPS states on the module $\hat{R}$. (BPS states having maximal supersymmetry correspond to connections with constant curvature. The results of [S] can be applied also to the case of BPS states having less supersymmetries .)

It was mentioned in CDS that in the case of two-dimensional tori Morita equivalence is related to $T$-duality in string theory. This remark leads to a conjecture that the group $S O(n, n \mid \mathbf{Z})$ that appears in the present paper is related to a corresponding group in $T$-duality.

It is clear that the relations (1) are unchanged if each $U_{j}$ is changed by some phase factor. One can use this fact to arrange in various ways $\mathrm{R} 4$ that there is a bicharacter $\gamma$ from $\mathbf{Z}^{n}$ to the circle group $\mathbf{T}$, and unitary elements $U_{x}$ of $A_{\theta}$ for each $x \in \mathbf{Z}^{n}$ such that

$$
U_{x} U_{y}=\gamma(x, y) U_{x+y}
$$

and such that if $e_{j}$ denotes the $j^{\text {th }}$ standard basis vector in $\mathbf{Z}^{n}$ and if $U_{j}=U_{e_{j}}$, then the relations (1) are satisfied. One useful choice for $\gamma$ is

$$
\gamma(x, y)=e((x \cdot \theta y) / 2)
$$

In any event we will have

$$
\gamma(x, y) \bar{\gamma}(y, x)=\rho(x, y)
$$

where $\rho$ is the skew-bicharacter defined by

$$
\rho(x, y)=\rho_{\theta}(x, y)=e(x \cdot \theta y) \text {. }
$$

We will use $\rho$ below.

One can consider also a smooth version of a noncommutative torus $[\mathrm{R} 4$, R5], which is the algebra $A_{\theta}^{\infty}$ consisting of formal series

$$
\sum c_{j_{1} \ldots j_{n}} U_{1}^{j_{1}} \ldots U_{n}^{j_{n}}
$$

where the collection of coefficients belongs to the Schwartz space $\mathcal{S}\left(\mathbf{Z}^{n}\right)$. One can characterize $A_{\theta}^{\infty}$ as the subalgebra of $A_{\theta}$ consisting of vectors which are smooth with respect to the natural action of the $n$-dimensional commutative Lie algebra on $A_{\theta}$; this action is given by ${ }^{*}$-derivations $\delta_{j}$ defined by the formula $\delta_{j} U_{j}=i U_{j}, \delta_{j} U_{k}=0$ if $j \neq k$. We will consider the noncommutative torus as a $C^{\star}$-algebra $A_{\theta}$; however, our results remain correct if $A_{\theta}$ is replaced with $A_{\theta}^{\infty}$. 
The notion of (strong) Morita equivalence of $C^{\star}$-algebra was introduced and analyzed in [R1, R3]. We will use the following constructive definition of Morita equivalence for the case of unital $C^{\star}$-algebras. Let us consider a finite projective right module $\mathcal{E}$ over a $C^{\star}$-algebra $A$ (i.e. a module that can be considered as a direct summand of a finite-dimensional free module over $A$.) The algebra $E n d_{A} \mathcal{E}$ of endomorphisms of $\mathcal{E}$ has a canonical structure as a $C^{\star}$-algebra. We say that a $C^{\star}$-algebra $A^{\prime}$ is (strongly) Morita equivalent to $A$ if it is isomorphic to $E n d_{A} \mathcal{E}$ for some finite projective module $\mathcal{E}$. (This is sufficient for our present purposes, but more generally one must require that the module is full [R3], which is automatic here.)

Morita equivalent $C^{\star}$-algebras share many important properties. (They have equivalent categories of modules, isomorphic $K$-groups, cyclic homology etc.)

Let us start our development with the definition of an action of the group $O(n, n \mid \mathbf{R})$ on the space $\mathcal{T}_{n}$ of real antisymmetric matrices. We consider $O(n, n \mid \mathbf{R})$ as a group of linear transformations of the space $\mathbf{R}^{2 n}$ preserving the quadratic form $x_{1} x_{n+1}+x_{2} x_{n+2}+\ldots+x_{n} x_{2 n}$. It is convenient to denote coordinates in $\mathbf{R}^{2 n}$ as $\left(a^{1}, \ldots, a^{n}, b_{1}, \ldots, b_{n}\right)$. Then the quadratic form on $\mathbf{R}^{2 n}$ can be written as $a^{i} b_{i}$. Accordingly, we will often write the elements of $O(n, n \mid \mathbf{R})$ in $2 \times 2$ block form

$$
g=\left(\begin{array}{cc}
A & B \\
C & D
\end{array}\right) .
$$

Here the blocks $A, B, C, D$ are $n \times n$ matrices which satisfy $A^{t} C+C^{t} A=0=$ $B^{t} D+D^{t} B, \quad A^{t} D+C^{t} B=1$, where ${ }^{t}$ denotes transpose. The action of $O(n, n \mid \mathbf{R})$ on the space $\mathcal{T}_{n}$ of antisymmetric matrices is defined by the formula

$$
\theta^{\prime}=(A \theta+B)(C \theta+D)^{-1} .
$$

We emphasize that this action is defined only on the dense subset of $\mathcal{T}_{n}$ where $(C \theta+D)$ is invertible.

We will consider the subgroup $S O(n, n \mid \mathbf{Z})$ of $O(n, n \mid \mathbf{R})$ consisting of matrices with integer entries and determinant +1 . In order to give an elegant formulation of our results, we let

$$
\mathcal{T}_{n}^{0}=\left\{\theta \in \mathcal{T}_{n}: g \theta \text { is defined for all } g \in S O(n, n \mid \mathbf{Z})\right\} .
$$

A simple calculation shows that $\mathcal{T}_{n}^{0}$ is carried into itself by the action of $S O(n, n \mid \mathbf{Z})$. But it is not so obvious how big $\mathcal{T}_{n}^{0}$ is. However, the considerations which we must go through anyway in order to prove our main theorem will give us a way of seeing that $\mathcal{T}_{n}^{0}$ is "big" in a suitable sense. ( In particular, it is dense in $\mathcal{T}_{n}$.)

Theorem. For $\theta \in \mathcal{T}_{n}^{0}$ and $g \in S O(n, n \mid \mathbf{Z})$ the noncommutative torus corresponding to $g \theta$ is Morita equivalent to the noncommutative torus corresponding to $\theta$. 
We do not know whether the converse holds, that is, whether if two $\theta$ 's give Morita equivalent noncommutative tori then these $\theta$ 's must be in the same orbit for the action of $S O(n, n \mid \mathbf{Z})$. When $n=2$ the converse does hold [R2], and the appearance here of $S O(n, n \mid \mathbf{Z})$ is a generalization of the appearance of $S L(2, \mathbf{Z})$ discovered in [R2]. In the case $n>2$ the converse statement is proved in [S] for a modified definition of Morita equivalence. We will comment on what one can say for $\theta$ 's which are not in $\mathcal{T}_{n}^{0}$ after the end of the proof of this theorem.

For the proof of our this theorem we now define a suitable set of generators for $S O(n, n \mid \mathbf{Z})$.

For every matrix $R \in G L(n \mid \mathbf{Z})$ we can define the transformation $\rho(R) \in$ $S O(n, n \mid \mathbf{Z})$ by the formula

$$
\tilde{a}^{i}=R_{j}^{i} a^{j}, \quad \tilde{b}_{i}=\left(R^{-1}\right)_{i}^{j} b_{j} .
$$

If $N$ is an anti-symmetric $n \times n$ matrix with integer entries $n_{i j}$ we can construct an element $\nu(N)$ of $S O(n, n \mid \mathbf{Z})$ as a transformation

$$
\tilde{a}^{i}=a^{i}+n^{i j} b_{j}, \quad \tilde{b}_{i}=b_{i}
$$

Finally, for every integer $k$ we define an element $\sigma_{k} \in O(n, n \mid \mathbf{Z})$ by the formula

$$
\begin{aligned}
& \tilde{a}^{i}=b_{i} \text { for } 1 \leq i \leq k, \quad \tilde{a}^{i}=a^{i} \text { for } k<i \leq n, \\
& \tilde{b}_{i}=a^{i} \text { for } 1 \leq i \leq k, \quad \tilde{b}_{i}=b_{i} \text { for } k<i \leq n .
\end{aligned}
$$

Notice that $\sigma_{k} \in S O(n, n \mid \mathbf{Z})$ only when $k$ is even. When $n$ is odd no element of $\mathcal{T}_{n}$ can be invertible, and so $\sigma_{n}$ will have no element of $\mathcal{T}_{n}$ in its domain.

Lemma. The elements $\rho(R), \nu(N)$, and the single element $\sigma_{2}$, together generate the group $S O(n, n \mid \mathbf{Z})$.

Proof. Let $H$ denote the group generated by these elements. Clearly $H \subseteq S O(n, n \mid \mathbf{Z})$. For any anti-symmetric $n \times n$ matrix $N$ let $\mu(N)$ denote the transformation

$$
\tilde{a}^{i}=a^{i}, \quad \tilde{b}_{i}=b_{i}+n_{i j} a^{j} .
$$

Now suppose that $N$ has its only non-zero (integer) entries in the top-left $2 \times 2$ block. Then it is easily verified that when $\nu(N)$ is conjugated by $\sigma_{2}$ one obtains exactly $\mu(N)$. Thus $\mu(N) \in H$. If we now conjugate $\mu(N)$ by $\rho(R)$ as $R$ ranges over all permutation matrices, we obtain all the $\mu(N)$ for all $N$ which have only 2 non-zero entries. But every anti-symmetric matrix is a sum of such $N$ 's, and the map $N \mapsto \mu(N)$ is a homomorphism from the additive group of integervalued anti-symmetric matrices into $S O(n, n \mid \mathbf{Z})$. Thus $H$ contains all $\mu(N)$ for all such matrices $N$.

Let $E O(n, n \mid \mathbf{Z})$ denote the subgroup of $S O(n, n \mid \mathbf{Z})$ generated by all the $\mu(N)$ 's and $\nu(N)$ 's, together with those $\rho(R)$ for which $R$ is an elementary matrix (i.e. is $I_{n}$ plus only one off-diagonal integer non-zero entry). Then 
$E O(n, n \mid \mathbf{Z}) \subseteq H$. Notice that $O(1,1 \mid \mathbf{Z})$ is a 4-element group, with subgroup $S O(1,1 \mid \mathbf{Z})$ of order 2 . When this observation is used in theorem 5.5.3 of [HO], one finds that $E O(n, n \mid \mathbf{Z})$ is a normal subgroup of $S O(n, n \mid \mathbf{Z})$ of index at most 2 , and that $E O(n, n \mid \mathbf{Z})$ together with $\rho(U) \oplus I_{n-2}$ generates $S O(n, n \mid \mathbf{Z})$, where $U$ is the non-identity element of $S O(1,1 \mid \mathbf{Z})$. (See the remark near the bottom of page 232 of $[\mathrm{HO}]$.) Since this element is in $H$, it follows that $H=S O(n, n \mid \mathbf{Z})$ as desired. (From theorem 7.2.23 of [HO] one sees that $E O(n, n \mid \mathbf{Z})$ is not itself all of $S O(n, n \mid \mathbf{Z})$.)

We now show that $\mathcal{T}_{n}^{0}$ is "big". Let $G$ denote the subgroup of $S O(n, n \mid \mathbf{Z})$ generated by the $\rho(R)$ 's and the $\nu(N)$ 's, so that it consists exactly of the $g \in$ $S O(n, n \mid \mathbf{Z})$ for which $C=0$. It is clear that $G$ acts on all of $\mathcal{T}_{n}$. Note that the action of $\sigma=\sigma_{2}$ is defined on $\theta \in \mathcal{T}_{n}$ exactly if the top-left $2 \times 2$ block of $\theta$ is non-zero, and so invertible. Let $U$ denote the set of such $\theta$ 's. Then $U$ is an open dense subset of $\mathcal{T}_{n}$, which is carried onto itself by $\sigma$ and contains $\mathcal{T}_{n}^{0}$. Let

$$
V_{1}=\bigcap\{g U: g \in S O(n, n \mid \mathbf{Z})\} .
$$

Since $S O(n, n \mid \mathbf{Z})$ is countable, Baire's theorem tells us that $V_{1}$ is a dense subset of $\mathcal{T}_{n}$ of the "second category" (that is, it is not the countable union of nowheredense sets). Clearly $V_{1}$ is $G$-invariant and contains $\mathcal{T}_{n}^{0}$. Let $U_{1}=\sigma\left(V_{1}\right)$. Then let

$$
V_{2}=\bigcap\left\{g U_{1}: g \in S O(n, n \mid \mathbf{Z})\right\} \cap V_{1},
$$

and let $U_{2}=\sigma\left(V_{2}\right)$. We continue inductively to define $V_{k}$ for all $k>0$. Each $V_{k}$ is $G$-invariant and contains $\mathcal{T}_{n}^{0}$. Set $\mathcal{T}_{n}^{1}=\bigcap^{\infty} V_{k}$. Again $\mathcal{T}_{n}^{1}$ is dense of the second category, so is "big". Clearly $\mathcal{T}_{n}^{1}$ is $G$-invariant and contains $\mathcal{T}_{n}^{0}$. But if $\theta \in \mathcal{T}_{n}^{1}$, then $\theta \in V_{k+1} \subseteq U_{k}$ for each $k$, so that $\sigma(\theta) \in V_{k}$. Thus $\sigma(\theta) \in \mathcal{T}_{n}^{1}$, and so $\sigma$ carries $\mathcal{T}_{n}^{1}$ into itself. In view of the Lemma, $S O(n, n \mid \mathbf{Z})$ has a fully-defined action on $\mathcal{T}_{n}^{1}$, carrying $\mathcal{T}_{n}^{1}$ into itself. Since $\mathcal{T}_{n}^{1}$ contains $\mathcal{T}_{n}^{0}$, it follows from the definition of $\mathcal{T}_{n}^{0}$ that $\mathcal{T}_{n}^{1}=\mathcal{T}_{n}^{0}$. Thus $\mathcal{T}_{n}^{0}$ is "big" as desired.

It is clearly sufficient to prove the theorem just for the $g$ 's in the generating set for $S O(n, n \mid \mathbf{Z})$ given in the Lemma above. To analyze the case $g=\rho(R)$ we should check that the noncommutative torus determined by the matrix $\theta^{\prime}=$ $R \theta R^{t}$ is Morita equivalent to the torus corresponding to the matrix $\theta$. One can verify these tori are in fact isomorphic. This is seen by using the description given above of noncommutative tori in terms of bicharacters. The matrix $R \in$ $S L(n, \mathbf{Z})$ generates an automorphism of $\mathbf{Z}^{n}$. The isomorphism between $A_{\theta^{\prime}}$ and $A_{\theta}$ follows from the relation

$$
U_{R(x)} U_{R(y)}=e\left(\left(x \cdot \theta^{\prime} y\right) / 2\right) U_{R(x+y)} .
$$

Next, it is obvious that replacing $\theta_{i j}$ with $\theta_{i j}^{\prime}=\theta_{i j}+n_{i j}$, where $n_{i j}$ are integers, we do not change the commutation relations (1) at all. This means that $\theta$ and $\theta^{\prime}=\nu(N) \theta$ correspond to the same noncommutative torus. 
It remains to prove that $A_{\theta}$ is Morita equivalent to $A_{\theta^{\prime}}$ when $\theta^{\prime}=\sigma_{2} \theta$. We will in fact prove this for any $\sigma_{2 p}$, and any $\theta$ in its domain.

Proposition. Let $\theta \in \mathcal{T}_{n}$, and assume that the top left $2 p \times 2 p$ block of $\theta$ (which we will denote by $\theta_{11}$ ) is invertible. Write $\theta$ in block form as

$$
\theta=\left(\begin{array}{cc}
\theta_{11} & \theta_{12} \\
\theta_{21} & \theta_{22}
\end{array}\right)
$$

Let

$$
\theta^{\prime}=\sigma_{2 p}(\theta)=\left(\begin{array}{cc}
\theta_{11}^{-1} & -\theta_{11}^{-1} \theta_{12} \\
\theta_{21} \theta_{11}^{-1} & \theta_{22}-\theta_{21} \theta_{11}^{-1} \theta_{12}
\end{array}\right) .
$$

Then the non-commutative torus for $\theta^{\prime}$ is Morita equivalent to that for $\theta$.

Proof. For the proof we use the construction of projective modules given in [R4]. To make the argument more transparant, we use ordering conventions which are especially adapted to our present situation. These conventions are slightly different from those used in [R4], but they only affect matters of orientation. Since we will not use the apparatus of connections and Chern character developed in [R4], this difference will have no effect on our present considerations.

Let

$$
J_{o}=\left(\begin{array}{cc}
0 & I_{p} \\
-I_{p} & 0
\end{array}\right) .
$$

Then $-\theta_{11}$ is similar to $J_{o}$, and so we can choose an invertible matrix, $T_{11}$, such that $T_{11}^{t} J_{o} T_{11}=-\theta_{11}$. Set $T_{13}=\theta_{12}^{t}$. Set $q=n-2 p$ and let $T_{32}$ be any $q \times q$ matrix such that $\theta_{22}=T_{32}^{t}-T_{32}$. For example, $T_{32}$ can be the part of $\theta_{22}$ above the main diagonal, with 0's below, or alternatively it can be $-\theta_{22} / 2$.

Set

$$
T=\left(\begin{array}{cc}
T_{11} & 0 \\
0 & I_{q} \\
T_{31} & T_{32}
\end{array}\right),
$$

a matrix of size $(n+q) \times n$. Also set

$$
J=\left(\begin{array}{ccc}
J_{o} & 0 & 0 \\
0 & 0 & I_{q} \\
0 & -I_{q} & 0
\end{array}\right),
$$

a square matrix of size $n+q$, which is for us a convenient way to write the standard symplectic matrix. A routine calculation shows that $T^{t} J T=-\theta$. (The minus sign is included because, as required by the definition of Morita equivalence, we will construct a right module. If one arranges for $+\theta$ here, then 
there will be unpleasant signs in the final formula.) Following the notation in [R], we let

$$
\tilde{T}=\left(\begin{array}{cc}
T_{11} & 0 \\
0 & I_{q}
\end{array}\right)
$$

and we note that $\tilde{T}$ is invertible. Note also that as a linear transformation, $T$ carries $\mathbf{Z}^{2 p} \times \mathbf{Z}^{q}$ into $\mathbf{R}^{2 p} \times \mathbf{Z}^{q} \times \mathbf{R}^{q}$. Thus $T$ satisfies the conditions in definition 4.1 of [R4 for being an "embedding" map. This means, as is anyway clear, that when we view $T$ as a homomorphism from $\mathbf{Z}^{n}$ into $G=\mathbf{R}^{2 p} \times \mathbf{Z}^{q} \times \mathbf{T}^{q}$, then the range of $T$ is a lattice in $G$, which we will denote by $D$. In what follows, our notation will not distinguish between elements of $\mathbf{R}^{2 p} \times \mathbf{Z}^{q} \times \mathbf{R}^{q}$ and their images in $G$.

We view $G$ as $M \times \hat{M}$ with $M=\mathbf{R}^{p} \times \mathbf{Z}^{q}$, where denotes "dual group". From this decomposition $G$ carries a canonical cocycle, $\beta$, called the "Heisenberg cocycle" in [R4], and defined by

$$
\beta((m, s),(n, t))=\langle m, t\rangle,
$$

where here $\langle$,$\rangle denotes the pairing between M$ and $\hat{M}$. The corresponding skew cocycle, $\rho$, is defined by

$$
\rho((m, s),(n, t))=\langle m, t\rangle \overline{\langle n, s\rangle} .
$$

It is clear that (after rearranging the order of the factors) we have exactly $\rho(x, y)=e(x \cdot J y)$ for the $J$ defined above. Because $T^{t} J T=-\theta$, the restriction of $\rho$ to $D$ and so to $\mathbf{Z}^{n}$ is exactly $(x, y) \mapsto e(-x \cdot \theta y)$. Except for a complex conjugation, this is exactly the $\rho$ defined much earlier on $\mathbf{Z}^{n}$.

The restriction of $\bar{\beta}$ to $D$ will then be a cocycle whose corresponding skew cocycle is exactly the restriction of $\rho$ to $D$, and so to $\mathbf{Z}^{n}$. Thus $\bar{\beta}$ is an instance of the cocycle $\gamma$ of equation (2) above. Consequently, when we let $A=\mathcal{S}\left(\mathbf{Z}^{n}, \bar{\beta}\right)$, the space of Schwartz functions on $\mathbf{Z}^{n}$ with convolution twisted by the cocycle $\bar{\beta}$, this algebra, or rather its $C^{\star}$-algebra completion, is (isomorphic to) our noncommutative torus $A_{\theta}$.

Because of the minus sign in $e(-x \cdot \theta y), A$ has a natural right action on the space $\mathcal{S}(M)$ of Schwartz functions on $M$, defined by a twisted convolution indicated shortly after the proof of proposition 2.9 of [R4] (except that there $D^{\perp}$ plays the role of our $D$ ). We do not need the formula for this action because what we really need is a description of the endomorphism algebra of this right $A$-module, since it is this endomorphism algebra which is Morita equivalent to $A$ via this module. But by applying proposition 3.2 of [R] we see that this endomorphism algebra is exactly $\mathcal{S}\left(D^{\perp}, \beta\right)$ where

$$
D^{\perp}=\{w \in G: \rho(w, z)=1 \text { for all } z \in D\},
$$

and then we restrict $\beta$ to $D^{\perp}$. This restricted $\beta$ will, of course, have the restricted $\rho$ as its corresponding skew cocycle. 
Thus we need to determine $D^{\perp}$. More specifically, we need to describe $D^{\perp}$ as the image of $\mathbf{Z}^{n}$ under some embedding map, so that we can calculate the anti-symmetric matrix $\theta^{\prime}$ which on $\mathbf{Z}^{n}$ gives the cocycle corresponding to the restriction of $\rho$ to $D^{\perp}$. To obtain the answer specified in the statement of the theorem, we need to choose this embedding map quite carefully. We proceed as follows.

Given $x \in G$, it will be in $D^{\perp}$ exactly if $x \cdot J T z \in \mathbf{Z}$ for all $z \in \mathbf{Z}^{n}$, that is, exactly if $T^{t} J x \in \mathbf{Z}^{n}$. We want a natural isomorphism from $\mathbf{Z}^{n}$ to this $D^{\perp}$. To see how to obtain one, let

$$
\bar{T}=\left(\begin{array}{ccc}
T_{11} & 0 & 0 \\
0 & I_{q} & 0 \\
T_{31} & T_{32} & I_{q}
\end{array}\right)
$$

a square matrix of size $n+q$ which is clearly invertible. It is easily checked that $T^{t} J x \in \mathbf{Z}^{n}$ exactly if $\bar{T}^{t} J x \in \mathbf{Z}^{n+q}$. Since $\bar{T}$ is invertible, as is $J$, this means that

$$
D^{\perp}=\left(\bar{T}^{t} J\right)^{-1}\left(\mathbf{Z}^{n+q}\right),
$$

viewed in $G$. But a routine calculation shows that

$$
\left(\bar{T}^{t} J\right)^{-1}=\left(\begin{array}{ccc}
-J_{o}\left(T_{11}^{t}\right)^{-1} & 0 & J_{o}\left(T_{11}^{t}\right)^{-1} T_{31}^{t} \\
0 & 0 & -I \\
0 & I & -T_{23}^{t}
\end{array}\right) .
$$

It is clear from this that

$$
\left(\bar{T}^{t} J\right)^{-1}\left(0 \times \mathbf{Z}^{q} \times 0\right)=0 \times 0 \times \mathbf{Z}^{q},
$$

which is 0 in $G=\mathbf{R}^{2 p} \times \mathbf{Z}^{q} \times \mathbf{T}^{q}$. Thus we can omit the second column of $\left(\bar{T}^{t} J\right)^{-1}$. This gives us, after we omit an inessential sign,

$$
S=\left(\begin{array}{cc}
J_{o}\left(T_{11}^{t}\right)^{-1} & -J_{o}\left(T_{11}^{t}\right)^{-1} T_{31}^{t} \\
0 & I \\
0 & T_{23}^{t}
\end{array}\right)
$$

This is the desired embedding map giving an isomorphism from $\mathbf{Z}^{n}$ onto $D^{\perp}$.

To conclude, we must calculate the matrix for the cocycle on $\mathbf{Z}^{n}$ coming from $\rho$ via $S$. But a routine calculation shows that $S^{t} J S=\sigma_{2 p}(\theta)$ whose matrix is given in the statement of the proposition.

We mention that F. Boca has pointed out to us that the case of the above proposition for $\sigma_{2}$ is implicit in calculations occurring shortly before lemma 2.1 of B], upon specialization of parameters. See also the remarks following lemma 2.6 of $\| \mathrm{B}$.

Let us make some comments about the proof of our theorem, as it applies to $\theta$ 's which are not in $\mathcal{T}_{n}^{0}$. We notice that each of our generators for $S O(n, n \mid \mathbf{Z})$ 
is defined on an open dense subset of $\mathcal{T}_{n}$. Let us suppose that $g \in S O(n, n \mid \mathbf{Z})$ is represented as $g_{1}, \ldots, g_{n}$ where $g_{i}$ are from among these generators. It is clear that we can find a dense open subset $\mathcal{T}^{g}$ of $\mathcal{T}_{n}$ such that $g_{i}$ is well defined on $g_{i+1} \ldots g_{n} \mathcal{T}^{g}$ for each $i$. It follows from our consideration that for $\theta \in \mathcal{T}^{g}$ the torus $\mathcal{A}_{g \theta}$ is Morita equivalent to $\mathcal{A}_{\theta}$.

Let us suppose that $\theta$ is in $\mathcal{T}_{n}$ but not in $\mathcal{T}^{g}$, that $g \in S O(n, n \mid \mathbf{Z})$, and that $C \theta+D$ is invertible, so that $g \theta$ is defined. One can conjecture that in this case the noncommutative torus for $g \theta$ is also Morita equivalent to that for $\theta$. We were not able to prove this conjecture.

\section{Appendix.}

One can define the action of $O(n, n \mid \mathbf{R})$ on $\mathcal{T}_{n}$ by considering a (non-linear) embedding of $\mathcal{T}_{n}$ into the Grassmann algebra $\mathcal{F}_{n}$ with generators $a^{1}, \ldots, a^{n}$. To every $\theta \in \mathcal{T}_{n}$ we assign the element

$$
\hat{\theta}=\exp \left(\frac{1}{2} a^{i} \theta_{i j} a^{j}\right) \in \mathcal{F}_{n}
$$

Let us introduce operators $\hat{a}^{j}$ and $\hat{b}_{k}$ transforming $\omega \in \mathcal{F}_{n}$ into $a^{j} \omega$ and $\frac{\partial \omega}{\partial a^{k}}$ respectively. (One can consider $\mathcal{F}_{n}$ as a Fock space; then these operators are creation and annihilation operators.) The operators $\hat{a}^{j} \hat{b}_{k}$ determine an irreducible representation of the Clifford algebra; in other words, they obey the canonical anticommutation relations: $\left[\hat{a}^{j}, \hat{a}^{k}\right]_{+}=\left[\hat{b}_{j}, \hat{b}_{k}\right]_{+}=0, \quad\left[\hat{a}^{j}, \hat{b}_{k}\right]_{+}=\delta_{k}^{j}$. Automorphisms of the Clifford algebra (linear canonical transformations) constitute a group, isomorphic to $O(n, n \mid \mathbf{R})$. We obtain a projective action of $O(n, n \mid \mathbf{R})$ on $\mathcal{F}_{n}$ by assigning to every automorphism $\alpha$ an operator $U_{\alpha}: \mathcal{F}_{n} \rightarrow \mathcal{F}_{n}$ by the formula $\alpha(a) \omega=U_{\alpha}(a \omega) U_{\alpha}^{-1}$, where $a$ is an arbitrary element of Clifford algebra. Restricting this action to $\mathcal{T}_{n} \subset \mathcal{F}_{n}$ we obtain the action (3). Indeed, the element $\hat{\theta}$ satisfies the equation

$$
\hat{b}_{i} \hat{\theta}=\left(\theta_{i j} \hat{a} j\right) \hat{\theta} .
$$

If

$$
\begin{gathered}
\alpha\left(\hat{a}^{i}\right)=A_{j}^{i} \hat{a}^{j}+B^{i j} \hat{b}_{j} \\
\alpha\left(\hat{b}_{i}\right)=C_{i j} \hat{a}^{j}+D_{i}^{j} \hat{b}_{j},
\end{gathered}
$$

then $\hat{\theta}^{\prime}=U_{\alpha} \hat{\theta}$ obeys

$$
\left(c_{i j} \hat{a}^{j}+D_{i}^{j} \hat{b}_{j}\right) \hat{\theta}^{\prime}=\left(\theta_{i k} A_{j}^{k} \hat{a}^{j}+\theta_{i k} B^{k j} \hat{b}_{j}\right) \hat{\theta}^{\prime} .
$$

In the case when the matrix $D_{i}^{j}=\theta_{i k} B^{k j}$ is invertible one can find $\hat{\theta}^{\prime}$ in the form $\exp \left(\frac{1}{2} a^{i} \theta_{i j}^{\prime} a^{j}\right)$. The expression for $\theta^{\prime}$ coincides with (3).

The description of the action of $O(n, n \mid \mathbf{R})$ in terms of the Grassmann algebra is useful if we would like to relate our theorem to known results about the $K$ groups and the cyclic homology of noncommutative tori. 
Let us consider the Grassmann algebra $\mathcal{F}_{n}^{\star}$ dual to the Grassmann algebra $\mathcal{F}_{n}$, and the integral lattice $\mathcal{F}_{n}^{\star}(\mathbf{Z})$ in $\mathcal{F}_{n}^{\star}$. The group $K_{0}\left(A_{\theta}\right)$ can be identified with the even part $\Lambda^{\text {even }}$ of this lattice and the group $K_{1}\left(A_{\theta}\right)$ can be identified with the odd part of it $[\mathrm{E}$. (Recall that a Grassmann algebra has a natural $\mathbf{Z}_{2}$-grading.) The action of $S O(n, n \mid \mathbf{R})$ on $\mathcal{F}_{n}$ induces an action of this group on $\mathcal{F}_{n}^{\star}$. The integral lattice in $\mathcal{F}_{n}^{\star}$ is carried into itself by the action of $S O(n, n \mid \mathbf{Z}) \subset$ $S O(n, n \mid \mathbf{R})$. Therefore we obtain an action of $S O(n, n \mid \mathbf{Z})$ on $K_{0}$ and on $K_{1}$. The canonical trace $\tau$ on $A_{\theta}$ determines a group homomorphism $\hat{\tau}$ of $K_{0}\left(A_{\theta}\right)$ into $\mathbf{R}$; if an element of $K_{0}\left(A_{\theta}\right)$ is represented by $x \in \Lambda^{\text {even }}$ then $\hat{\tau}(x)$ can be calculated as the scalar product $\langle\hat{\theta}, x\rangle$ where $\hat{\theta}$ is defined by the formula (5). Taking into account that $K$-groups of Morita equivalent tori coincide, we obtain a complete agreement between this expression for $\hat{\tau}$ and our theorem ( see [S] for more details).

It is interesting to notice that the embedding of $\mathcal{T}_{n}$ into $\mathcal{F}_{n}$ described above can be used to define a natural completion of $\mathcal{T}_{n}$. Namely, we should consider the closure $\overline{\mathcal{T}}_{n}$ of $\mathcal{T}_{n}$ embedded in $\mathcal{F}_{n}$. The projective action of $O(n, n \mid \mathbf{R})$ on $\mathcal{F}_{n}$ induces a projective action of this group on $\overline{\mathcal{T}}_{n}$. (Let us emphasize that there is no ambiguity in the action of $O(n, n \mid \mathbf{R})$ on $\mathcal{T}_{n}$.)

\section{Acknowledgements:}

We would like to thank M. Ratner, G. Prasad, and D. G. James for helpful comments about $O(n, n \mid \mathbf{Z})$. We thank F. Boca for pointing out an incorrect assertion in a previous version of this paper.

\section{References}

[B] Boca, F. P., The structure of higher-dimensional noncommutative tori and metric diophantine approximation, J. Reine Angew. Math. 492 (1997), 179-219.

[CDS] Connes, A., Douglas, M. R. and Schwarz A., Noncommutative Geometry and Matrix Theory: Compactification on Tori, hepth/9711162.

[DH] Douglas, M. and Hull, C., D-branes and non-commutative geometry, hep-th/9711165.

[E] Elliott, G. A., On the K-theory of the $C^{*}$-algebras generated by a projective representation of a torsion-free discrete abelian group, in "Operator Algebras and Group Representations" 157-184, Pitman, London, 1984.

[HO] Hahn, A, J. and O'Meara, O. T., "The Classical Groups and K-theory" Springer-Verlag, 1989. 
[R1] Rieffel, M. A., Induced representations of $C^{*}$-algebras, Advances Math. 13 (1974), 176-257.

[R2] Rieffel, M. A., $C^{*}$-algebras associated with irrational rotations, Pacific J. Math. 93 (1981), 415-429.

[R3] Rieffel, M. A., Morita equivalence for operator algebras, in "Operator Algebras and Applications" (R. V. Kadison, ed.) Proc. symp. Pure Math. 38, Amer. Math. Soc., Providence, 1982.

[R4] Rieffel, M. A., Projective modules over higher-dimensional noncommutative tori, Canadian J. Math. 40 (1988), 257-338.

[R5] Rieffel, M. A., Non-commutative tori - a case study of noncommutative differentiable manifolds, Contemporary Math. 105 (1990), 191-211.

[S] Schwarz, A.,Morita equivalence and duality, in preparation.

[T] Kawano, T. and Okuyama, K., Matrix Theory on Noncommutative Torus, hep-th/9803044

Cheu, Y.-K. E. and Krogh, M., Noncommutative Geometry from 0-branes in a Background B-field, hep-th/9803031

Berkooz, M., Non-local Field Theories and the Non-commutative Torus, hep-th/9802069

Nekrasov, N. and Schwarz, A., Instantons on noncommutative $R^{4}$, and $(\mathbf{2 , 0})$ superconformal six dimensional theory, hep-th/9802068 Li, M., Comments on Supersymmetric Yang-Mills Theory on a Noncommutative Torus, hep-th/9802052

Taylor, W., Lectures on D-branes, Gauge Theory and M(atrices), hep-th/9801182

Casalbuoni, R., Algebraic treatment of compactification on noncommutative tori, hep-th/9801170

Ho, P.-M. and Wu, Y.-S., Noncommutative Gauge Theories in Matrix Theory, hep-th/9801147

Krogh, M., A Matrix Model for Heterotic $\operatorname{Spin}(32) / Z_{2}$ and Type I String Theory, hep-th/9801034

Ho, P.-M., Wu, Y.-Y., and Wu, Y.-S., Towards a Noncommutative Geometric Approach to Matrix Compactification, hepth/9712201

Leigh, R. G. and Rozali, M., A Note on Six-Dimensional Gauge Theories, hep-th/9712168

Aharony, O., Berkooz, M. and Seiberg, N., Light-Cone Description of $(2,0)$ Superconformal Theories in Six Dimensions, hepth/9712117

Obers, N. A., Pioline, B. and Rabinovici, E., M-Theory and U-duality 
on $T^{d}$ with Gauge Backgrounds, hep-th/9712084

Hull, C. M., U-Duality and BPS Spectrum of Super Yang-Mills Theory and M-Theory, hep-th/9712075

Blau, M. and O'Laughlin, M., Aspects of U-Duality in Matrix Theory, hep-th/9712047

e-mail: rieffel@math.berkeley.edu

e-mail: schwarz@math.ucdavis.edu 